\title{
Biofilms: United We Stand, Divided We Fall
}

\author{
Jasvinder Kaur $\cdot$ Neha Niharika $\cdot$ Pushp Lata $\cdot$ \\ Rup Lal
}

Published online: 26 March 2014

(C) Association of Microbiologists of India 2014

Bacteria have been into existence since the dawn of time. From the discovery of bacteria to the heyday of antibiotic discovery, our view of bacteria has shifted dramatically. It is now widely accepted that microbes rarely survive as solitary cells, but rather grow as biofilms. A biofilm is a surface-associated population of microbes that is embedded in a cement of extracellular compounds. This cement is known as matrix and synonymously as extracellular polymeric substance [1].

Biofilms can contain different types of microorganisms and are ubiquitous. Recently, viral biofilms have come into picture with the finding that in the case of HTLV-I, biofilms may be a major mechanism of its propagation and transmission. HTLV-1 infects 15-20 million people worldwide. Although most of the infected individuals are asymptomatic, 5-10\% develop $\mathrm{T}$ cell leukemia or inflammatory syndrome such as HTLV-1-associated myelopathy. Extracellular viral assemblies account for over $80 \%$ of the infectious capacity of HTLV-1-infected cells. Both collagen and fibronectin are overexpressed in HTLV- 1 infected cells, collagen is enriched in viral assemblies but randomly distributed on uninfected cells. Although heparin washes do not completely remove extracellular viral assemblies, but the infectious capacity of cells is markedly reduced. Cell supernatant obtained after cell washes is infectious, although much less efficient than infected cells, indicating

This is a republication of the original article that was only published in print in the back matter of the AMI-produced, Indian version of the journal (vol. 50: 364-365 issue no. 3, September 2010). For citation purposes, please refer to the current version of the article.

J. Kaur $\cdot$ N. Niharika $\cdot$ P. Lata $\cdot$ R. Lal $(\bowtie)$

Department of Zoology, University of Delhi,

New Delhi 110 007, India

e-mail: ruplal@gmail.com that the integrity of extracellular viral assemblies and their transfer at cell contacts are key for HTLV-1 transmission. It is likely that other viruses have also developed transmission strategies based on similar biofilm-like viral assemblies. As extracellular structures of a particular composition, viral biofilms might be potential targets for future antiviral therapy [1].

Apart from this, biofilms have been a cause of concern as microbial biofilms on surfaces cost billions of dollars yearly in equipment damage, product contamination, energy losses and medical infections. Nevertheless, biofilms can also be harnessed for constructive purposes like bioremediation of hazardous materials and waste sites, biofilteration of industrial waste water and formation of natural biological barriers to protect soil and ground water from contamination. They can also be seen as a source of bioactive agents as bacteria in biofilms produce such chemical weapons which individual bacteria cannot produce like violecin pigment which bacteria in marine biofilms release to combat the enemy (paralysing the attacker).

Microbes are smarter than we thought and combating biofilms is a hard nut to crack as biofilms can require $100-1,000$ times the concentration of an antibiotic to control an infection which is medically impractical to administer. Also, repeated use of anti-microbial agents can cause bacteria to develop increased resistance. This virtue is imparted by the 'matrix' which prevents drugs and stress from penetrating the biofilm as well as holds the cells together. Matrix also provides a means by which bacteria within a biofilm can communicate amongst themselves by producing specific signals and arousing an attack, a phenomenon known as quorum sensing, jamming these signals might provide a way to fight back.

So, the solution to the problem lies in divide and rule policy. Enzymes such as Dispersin B and deoxyribonuclease 
degrade the matrix which holds the cells together and may play a role as anti-biofilm agents. Genes like ZapI have been found which have negative effect on matrix formation [2]. Some bacteriophages produce polysaccharide depolymerases that have the potential to degrade biofilm matrix [3].

Hence, the biofilm research should focus on bacteria in the biofilm rather than suspended bacteria in culture as the properties and the genetic regulation of the two are different since bacteria in biofilm behave differently from planktonic cells. Achieving the task may seem difficult but not impossible. The day shall come soon when the concept of biofilms will only be utilized for the benefit of mankind.

\section{References}

1. Pais-Correia AM, Sachse M, Guadagnini S, Robbiati V, Lasserre R, Gessain A, Gout O, Alcover A, Thoulouze MI (2010) Biofilmlike extracellular viral assemblies mediate HTLV-1 cell-to-cell transmission at virological synapses. Nat Med 16:83-89

2. Nobile CJ, Nett JE, Hernday AD, Homann OR, Deneault JS, Nantel A, Andes DR, Johnson AD, Mitchell AP (2009) Biofilm matrix regulation by Candida albicans Zap1. PLoS Biol 7:1-15

3. Donlan RM (2009) Preventing biofilms of clinically relevant organisms using bacteriophage. Trends Microbiol 17:66-72 